\title{
Andrographolide protects against endothelial dysfunction and inflammatory response in rats with coronary heart disease by regulating PPAR and NF-кB signaling pathways
}

\author{
Jia Shu ${ }^{1 \#}$, Ruizhen Huang ${ }^{2 \#}$, Ying Tian ${ }^{3 \#}$, Yanru Liu ${ }^{2}$, Rui Zhu ${ }^{2}$, Gang Shi ${ }^{4}$ \\ ${ }^{1}$ Functional Inspection Division, ${ }^{2}$ Department of Cardiovascular, ${ }^{3}$ Department of Urology Surgery, ${ }^{4}$ Department of Pharmacy Services, Hospital of \\ Chengdu University of Traditional Chinese Medicine, Chengdu, China \\ Contributions: (I) Conception and design: J Shu, R Huang, Y Tian, G Shi; (II) Administrative support: J Shu, Y Liu; (III) Provision of study materials \\ or patients: J Shu, R Huang, Y Liu, R Zhu; (IV) Collection and assembly of data: All authors; (V) Data analysis and interpretation: R Huang, Y Tian, \\ R Zhu, G Shi; (VI) Manuscript writing: All authors; (VII) Final approval of manuscript: All authors. \\ \#These authors contributed equally to the work and shared first authorship work. \\ Correspondence to: Gang Shi. Department of Pharmacy Services, Hospital of Chengdu University of Traditional Chinese Medicine, No. 39 Shi-er-qiao \\ Road, Chengdu, China. Email: shigangsssss@hotmail.com.
}

\begin{abstract}
Background: Andrographolide (Andro) is an active compound extracted from Andrographis, which has protective anti-inflammatory effects. But, its pathological role in coronary heart disease (CHD) is unclear, the aim of this study is to investigate the therapeutic effect of Andro in CHD and explore its potential mechanism.
\end{abstract}

Methods: Here, we established a mouse model of CHD, and rats were randomly divided into 5 groups $(\mathrm{n}=10)$ : sham, Andro (50 mg/kg), CHD, CHD + Andro (10 mg/kg), and CHD + Andro (50 mg/kg). HE staining was employed to evaluate the pathological changes of myocardial injury cardiac injury. The serum levels of total cholesterol (TC), triglycerides (TG), low-density lipoprotein cholesterol (LDL-C), highdensity lipoprotein cholesterol (HDL-C), nitric oxide (NO), TXA2, ET-1, and prostaglandin I2 (PGI2) were detected by ELISA assay. Myocardial inflammation and the interaction between Andro and PPAR- $\alpha / \mathrm{NF}-\kappa \mathrm{B}$ axis was measured using western blot.

Results: Compared with CHD groups, Andro preserved cardiac injury and decreased the levels of TC, TG, and LDL-C while increasing the level of HDL-C. In addition, Andro also reduced the levels of TNF- $\alpha$, MCP-1, hs-CRP and IL- $1 \beta$ by shifting the macrophage phenotype and attenuated the endothelial dysfunction by increasing the serum levels of ET-1 and TAX2 and decreasing the levels of NO and PGI2 in mice. Furthermore, Andro impeded cardiac apoptosis and inhibited the activation of PPAR $\alpha$ and NF- $\mathrm{BB}$ proteins.

Conclusions: Andro may represent a medicinal approach for assessing and treating CHD.

Keywords: Andrographolide; inflammation; coronary heart disease (CHD); PPAR; and NF-кB

Submitted Mar 22, 2020. Accepted for publication Jun 24, 2020.

doi: 10.21037/apm-20-960

View this article at: http://dx.doi.org/10.21037/apm-20-960

\section{Introduction}

Coronary artery disease (CAD) is one of the leading causes of morbidity and mortality worldwide (1). Despite tremendous progress in diagnosis and treatment, it remains an important public health issue. Especially in China, the incidence of $\mathrm{CAD}$ continues to rise, causing more than $40 \%$ of deaths (2). CAD is usually caused by atherosclerotic lesions and causes myocardial ischemia (3). Coronary heart disease (CHD) is a general term for coronary atherosclerotic lesions caused by multiple factors (4). As a 
Table 1 The details of antibodies used in this experiment

\begin{tabular}{|c|c|c|c|}
\hline Protein & $\begin{array}{l}\text { Molecular weight } \\
(\mathrm{kDa})\end{array}$ & $\begin{array}{l}\text { Dissolution } \\
\text { ratio }\end{array}$ & Source \\
\hline t-PA & 63 & $1 / 1,000$ & ab157469, Abcam \\
\hline PAl-1 & 45 & $1 / 500$ & ab66705, Abcam \\
\hline Caspase-3 & 34 & $1 / 500$ & ab13847, Abcam \\
\hline Bax & 21 & $1 / 1,000$ & ab32503, Abcam \\
\hline $\mathrm{Bcl}-2$ & 26 & $1 / 500$ & ab59348, Abcam \\
\hline PPAR $\alpha$ & 40 & $1 / 300$ & ab23673, Abcam \\
\hline p65 & 64 & $1 / 1,000$ & ab16502, Abcam \\
\hline p-p65 & 60 & $1 / 2,000$ & ab86299, Abcam \\
\hline $\mid \kappa B \alpha$ & 35 & $1 / 1,000$ & ab32518, Abcam \\
\hline$p-\mid \kappa B \alpha$ & 40 & $1 / 10,000$ & ab133462, Abcam \\
\hline$\beta$-actin & 42 & $1 / 5,000$ & ab179467, Abcam \\
\hline
\end{tabular}

common cardiovascular disease (CVD), CHD has become a major health problem for the general population in the past few decades. More specifically, CHD is a dynamic process of the interaction between endothelial dysfunction and inflammatory response (5). Its diagnosis and treatment methods are inconvenient, have low specificity, and are accompanied by complications such as organ failure, diabetes and depression. Therefore, a multi-target, multipath treatment is urgently needed.

Endothelium, a monolayer of endothelial cells, separates the vascular wall from circulation, regulates vascular tension, and maintains a homogenous balance of blood vessels (6). However, endothelial dysfunction limits the production of various vascular protective molecules. CVD begins with progressive impairment of endothelial function and integrity (7). Currently, there is little strong evidence supporting a link between endothelial dysfunction and the pathogenesis of CVD. Inflammation has been revealed to be one of the causes of CHD (8), and the expression of inflammatory factors such as IL- 6 , TNF- $\alpha$, and IL-1 in patients with CHD may be related to the pathogenesis of CHD. Previous reports suggest that suppressing inflammation helps treat CHD (9). If the internal environment of blood vessels is imbalanced, atherosclerotic plaques may be formed in the vasculature under the influence of inflammation and risk factors (10), leading to the occurrence of CHD (11).

Andrographis paniculata is a Chinese herbal medicine used to treat diseases such as laryngitis, diarrhea, and rheumatoid arthritis (12). Andrographolide (Andro) is a bicyclic diterpene lactone isolated from Andrographis, which exhibits anti-cancer (13), hepatocyte protection (14), and anti-inflammatory activity (15). It has been confirmed that Andro can improve endotoxemia in rats by inhibiting inducible nitric oxide (NO) synthase to reduce $\mathrm{NO}$ production (16). In addition, Xia et al. found that Andro covalently bound cysteine 62 of p50, blocking the NF-кBactivated inflammatory response (17).

Although the protective effect of Andro has been reported in vitro, its role in vivo, especially in CHD, is unclear. In this study, we evaluated the therapeutic role of Andro in a mouse model of CHD and its underlying mechanisms. We also investigated the effects of Andro on endothelial dysfunction and inflammation.

We present the following article in accordance with the ARRIVE reporting checklist (available at http://dx.doi. org/10.21037/apm-20-960).

\section{Methods}

\section{Main materials and chemicals}

Andrographolide (molecular formula: $\mathrm{C}_{20} \mathrm{H}_{30} \mathrm{O}_{5}$, molecular weight: 350.45$)$ was obtained from Shanghai Standard Technology Co., Ltd. (Shanghai, China). All antibodies were obtained from Abcam (Cambridge, UK) in Table 1.

\section{Animal model}

Healthy male C57BL/6 mice (8 weeks old) were obtained from Zhejiang Chinese Medicine University Laboratory Animal Research Center (Hangzhou, China). All mice were housed in a comfortable environment $\left(22{ }^{\circ} \mathrm{C}\right.$, humidity of $55 \%$ and light/dark cycle for $12 \mathrm{~h}$ ) with free access to food and water. All animal protocols were conducted in accordance with the institute guidelines for the care and use of animal, and approved by the ethics committees of Chengdu University of Traditional Chinese Medicine (No. SYXK-20200046). According to a previous description (18), a high-fat diet model of mouse CHD (19) was established. Mice were randomly divided into five groups $(\mathrm{n}=10)$ : sham, Andro (50 mg/kg), CHD, CHD + Andro (10 mg/kg), and CHD + Andro $(50 \mathrm{mg} / \mathrm{kg})$. The mouse model was established by intraperitoneal injection with vitamin D3 $(600,000 \mathrm{U} / \mathrm{kg})$, while other groups received an equal volume of sterile saline. Then, 42 days later, mice were sacrificed and heart tissues and serum samples were 
immediately collected and stored at $-80{ }^{\circ} \mathrm{C}$ for follow-up experiments.

\section{Hematoxylin and eosin (HE) staining}

In short, mouse cardiac tissues were fixed in $4 \%$ paraformaldehyde buffer for $2 \mathrm{~h}$. The specimens were continuously cut into $4 \mu \mathrm{m}$ thick slices after embedding in paraffin. Next, slices were stained with hematoxylin for $5 \mathrm{~min}$, and eosin for $3 \mathrm{~min}$. Finally, the slices were dehydrated and blocked for pathological diagnosis.

\section{Determination of blood lipid}

The levels of triglycerides (TG), total cholesterol (TC), low-density lipoprotein cholesterol (LDL-C) and highdensity lipoprotein cholesterol (HDL-C) in serum were measured using a fully automatic biochemical analyzer (BS-220, Mindray, Shenzhen, China) according to the commercially available kit instructions (Lai Er BioTech, Hefei, China), samples were transferred into a 96well microplate for colorimetric analysis of absorbance at $500 \mathrm{~nm}$.

\section{Enzyme-linked immunosorbent assay (ELISA) assay}

After andrographolide treatment as described above, serum was collected after centrifugation for $10 \mathrm{~min}\left(4^{\circ} \mathrm{C}, 2,000 \mathrm{~g}\right)$. Serum levels of related proteins were detected using enzyme-linked immunosorbent assay (ELISA) kits (MskBio, Wuhan, China). The operational steps were performed according to the manufacturer's instructions, and the results at $450 \mathrm{~nm}$ were recorded. The average absorbance values of repeated standard and samples were determined. By the best fitting curve, the sample concentration was calculated for data analysis.

\section{Flow cytometry}

After mice were sacrificed, the heart tissue was quickly removed and placed in ice-filled PBS. The heart tissue was minced and digested, and the mixture was centrifuged at $500 \mathrm{~g}$ for $5 \mathrm{~min}$ at $4^{\circ} \mathrm{C}$, then the supernatant was removed and the cells were resuspended in $1 \mathrm{~mL}$ of ice staining buffer. The cells were divided into three parts according to the cell count, and each part contained about $10^{6}$ live cells for flow cytometry. Next, the isolated cells were coupled with macrophage-conjugated primary antibodies on ice incubate for $30 \mathrm{~min}$. Subsequently, the cell samples were cleaned twice and then analyzed by a CytoFLEX flow cytometer (Beckman Coulter, CA, USA). F4-80 $/ \mathrm{CD}^{+}$ (pro-inflammatory) and $\mathrm{F} 4-80^{+} / \mathrm{CD}^{2} 06^{+}$(anti-inflammatory) as the macrophage phenotypes. APC-CY5.5 conjugated CD86, APC-CY7 conjugated CD206 and R-PE-CY5 conjugated $\mathrm{F} 4 / 80$.

\section{Immunobistochemistry}

Mouse heart tissue was fixed in $10 \%$ formalin solution. After embedding paraffin, the tissues were cut into $4 \mu \mathrm{m}$ thickness. Next, the sections are hydrated and endogenous peroxidase activity was blocked with hydrogen peroxide blockers. The antigen was repaired in citrate heated buffer for $10 \mathrm{~min}$, and then the sections were incubated with anti-caspase- 3 antibody overnight at $4{ }^{\circ} \mathrm{C}$. Next day, the second antibody was incubated at room temperature for 1 h. Samples were stained with a DAB kit (Sangon Biotech, shanghai, china). Finally, the sections were cleaned and the images were captured under a confocal microscope (Leica, Wetzlar, Germany).

\section{Western blotting}

Total protein were lysed with RIPA Lysis and Extraction Buffer (Thermo Fisher Scientific, Waltham, USA), incubated on ice for $15 \mathrm{~min}$, and centrifuged at $10,000 \mathrm{~g}$ for $10 \mathrm{~min}$. A bicinchoninic acid assay (BCA) kit (Abcam, Cambridge, UK) was used to measure the protein concentration. Afterwards, protein samples were split by $12 \%$ SDS-PAGE, then transferred to polyvinylidene difluoride (PVDF) (Thermo Fisher Scientific, Waltham, USA) membrane. Next, the membranes were blocked with $5 \%$ non-fat milk at $37^{\circ} \mathrm{C}$ for $1 \mathrm{~h}$, and subsequently incubated with the matching primary antibody at $4{ }^{\circ} \mathrm{C}$ overnight. $\beta$-actin was represented as the internal control. After being fully washed 3 times, the membranes were incubated with goat anti-rabbit antibody coupled with horseradish peroxidase (HRP) for $2 \mathrm{~h}$ at $37^{\circ} \mathrm{C}$. Finally, the protein blotting was visualized with enhanced chemiluminescence (ECL) reagent (Beyotime, Haimen, China).

\section{Statistical analyses}

All operations are repeated at least 3 times, and the results are represented as mean \pm SD (standard deviation). Data 

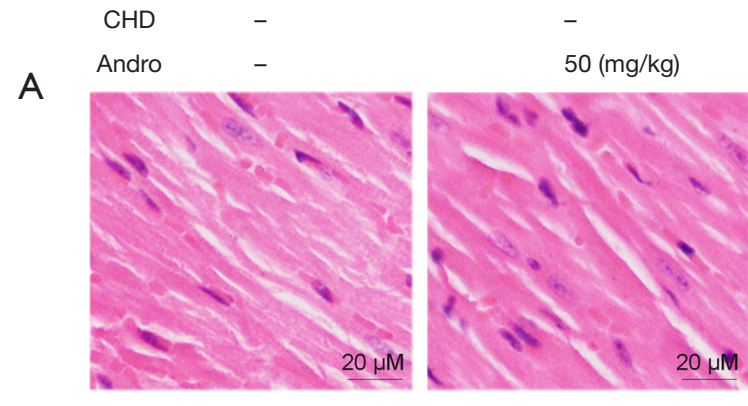

B
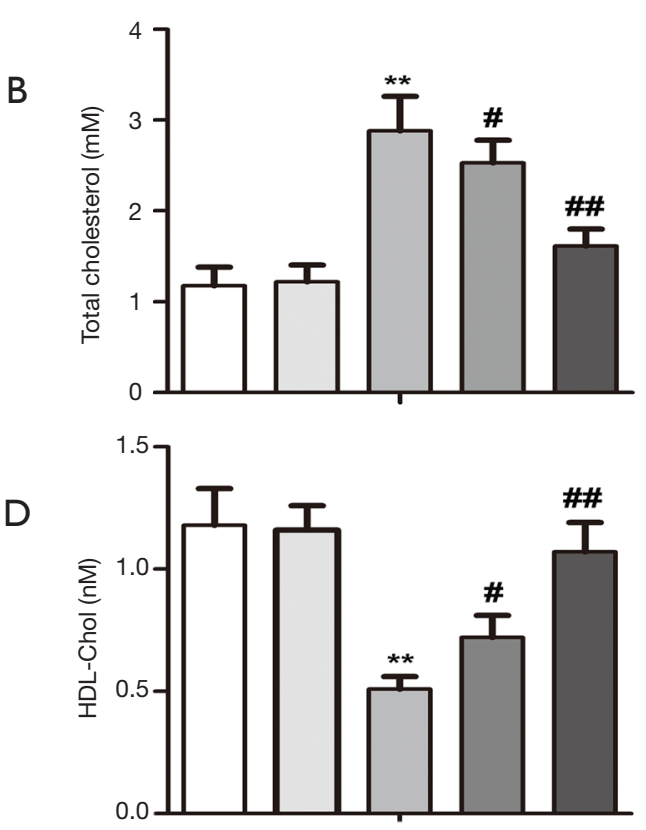
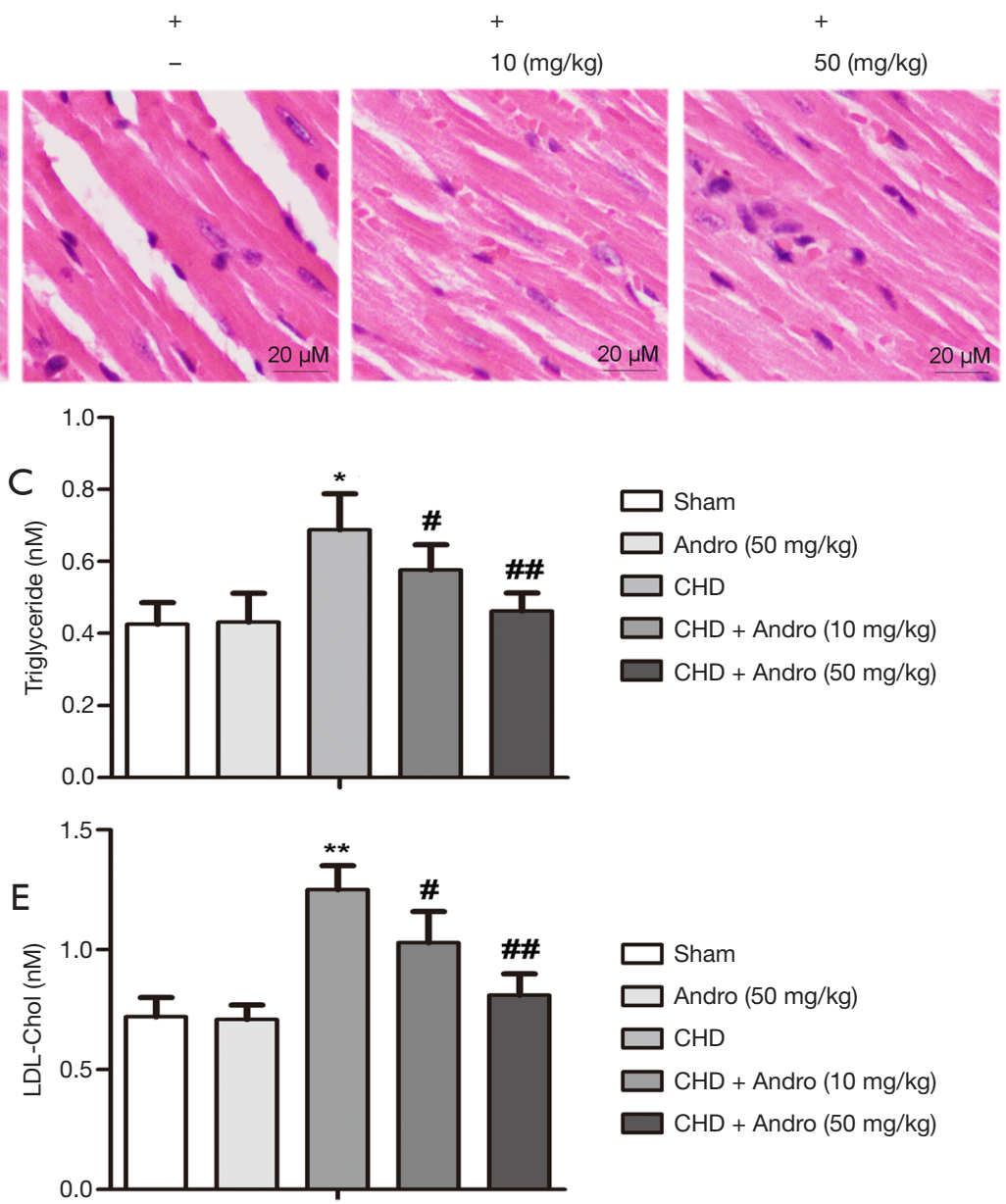

Figure 1 Effect of Andro on cardiac injury and serum lipid profiles. (A) HE staining of myocardial tissue; representative micrographs were magnified at 400x; (B,C,D,E) the serum contents of TC, TG, LDL-C, and HDL-C as detected by ELLSA assay. Data values are presented as mean $\pm \mathrm{SD} ; \mathrm{n}=10$. *, $\mathrm{P}<0.05,{ }^{* *}, \mathrm{P}<0.01$ vs. sham group; ${ }^{*}, \mathrm{P}<0.05,{ }^{\#}, \mathrm{P}<0.01$ vs. CHD group. Andro, andrographolide; TC, total cholesterol; TG, triglycerides; LDL-C, low-density lipoprotein cholesterol; HDL-C, high-density lipoprotein cholesterol; CHD, coronary heart disease.

were analyzed using SPSS 21.0 (IBM, Armonk, NY, USA). Comparisons between the two groups were performed using Student's $t$-test, while comparisons among multiple groups were conducted using one-way analysis of variance (ANOVA) followed by Dunnett's post hoc test. P values $<0.05$ were considered statistically significant.

\section{Results}

\section{Andrographolide preserved cardiac injury and improved the serum lipid profiles after CHD}

To investigate the effect of Andro on myocardium after $\mathrm{CHD}$, we observed the pathological changes of heart tissue by hematoxylin and eosin (HE) staining. As shown in Figure $1 A$, there was no significant difference of morphology and structure in the sham group. Whereas, cell edema, necrosis and neutrophil infiltration occurred in the CHD group. Excitingly, compared with the CHD-induced group, myocardial tissue damage was gradually repaired with Andro (10 and $50 \mathrm{mg} / \mathrm{kg}$ ). In addition, we tested the blood lipid profile (TC, TG, LDL-C, HDL-C) by ELISA assay, and the results showed that the serum contents of the TC, TG, and LDL-C in the CHD group were higher than those in the sham group, while HDL-C was lower, and there was no significant change in Andro $50 \mathrm{mg} / \mathrm{kg}$ (Figure 1B,C,D,E). Compared with the CHD group, the levels of TC, TG, and LDL-C in the CHD + Andro (10 and 

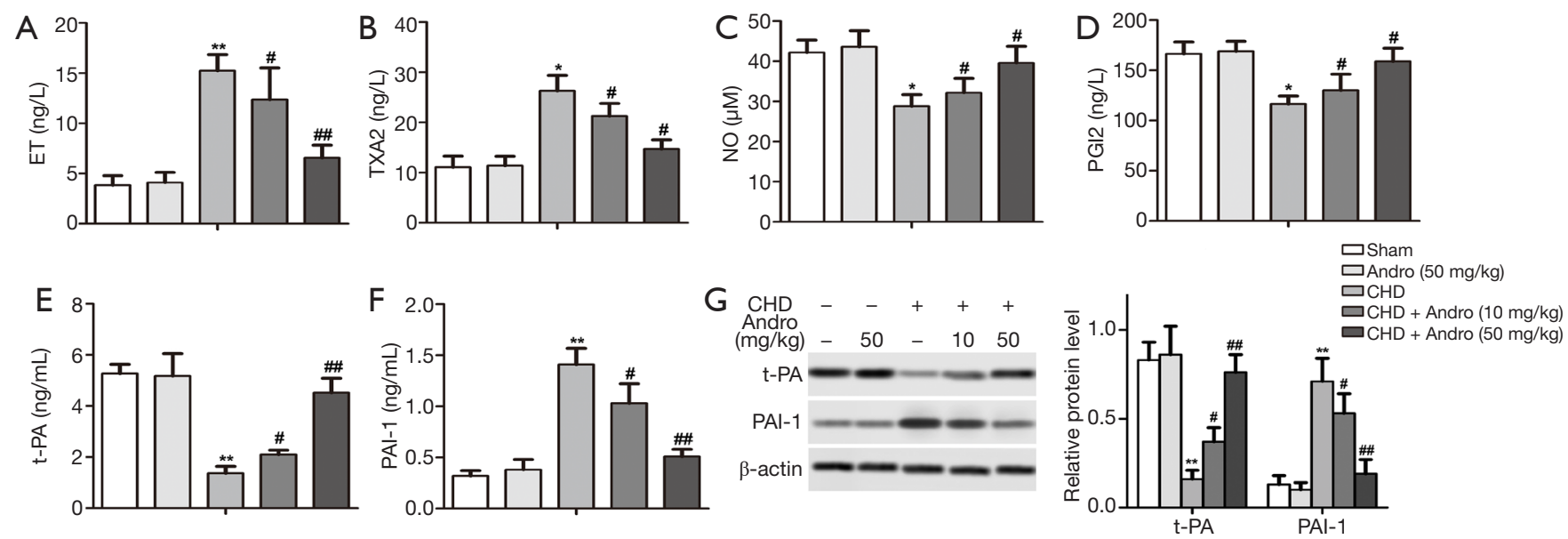

Figure 2 Effect of Andro on endothelial dysfunction and fibrinolytic activity. (A,B,C,D) The serum levels of NO, TXA2, ET-1, and PGI2,as detected by ELLSA assay; (E,F) the plasma levels of t-PA and PAI-1 as detected by ELISA assay; (G) the protein levels of t-PA and PAI-1 as detected by western blot. Data values are presented as mean $\pm \mathrm{SD} ; \mathrm{n}=10$. * $, \mathrm{P}<0.05,{ }^{* *}, \mathrm{P}<0.01$ vs. sham group; ${ }^{\#}, \mathrm{P}<0.05,{ }^{\# \#}, \mathrm{P}<0.01$ vs. $\mathrm{CHD}$ group. Andro, andrographolide; NO, nitric oxide; CHD, coronary heart disease.

$50 \mathrm{mg} / \mathrm{kg}$ ) groups were significantly decreased, while that of HDL-C was increased (Figure 1B,C,D,E). The results show that Andro could protect myocardial injury and normalize lipid disorder by improving blood lipid profiles.

\section{Andrographolide mitigated endothelial dysfunction and fibrinolytic activity after CHD}

Changes in NO and ET-1 can cause vascular endothelial dysfunction (20), and prostaglandin I2 (PGI2) and thromboxane A2 (TXA2) are 2 important active factors in the vascular system (21). Therefore, we tested NO, ET-1, PGI2, and TXA2 serum levels by ELLSA assay. As expected, compared with sham group, the levels of ET-1 (Figure $2 A$ ) and TAX2 (Figure 2B) were significantly increased in CHD-induced mouse serum, while the serum levels of $\mathrm{NO}$ (Figure 2C) and PGI2 (Figure 2D) were decreased. Interestingly, Andro treatment reversed this trend. The results of serum NO, ET-1, TXA2, and PGI2 indicated that Andro had a protective effect on vascular endothelial dysfunction caused by CHD. In addition, compared with the sham group, the level of t-PA was significantly decreased and PAI-1 was increased in the CHD group. Conversely, Andro (10 and $50 \mathrm{mg} / \mathrm{kg}$ ) increased the content of t-PA and decreased PAI-1, compared with the CHD group (Figure 2E,F). Western blot results of t-PA and PAI1 were consistent with ELLSA (Figure 2G), indicating that Andro improved fibrinolytic function and reduced blood coagulation.

\section{Andrographolide switched the macrophage phenotype and ameliorated myocardial inflammation}

Treatment with Andro (10 and $50 \mathrm{mg} / \mathrm{kg}$ ) significantly decreased $\mathrm{CD}^{2} 6^{+}$cells number and rose CD206 ${ }^{+}$cells number (Figure 3A). Notably, as shown in Figure 3B, compared with sham, the ratio of $\mathrm{CD} 86^{+} / \mathrm{CD} 206^{+}$was decreased in macrophages post Andro treatment. Previous reports have shown that inflammatory response is associated with the progression of CHD (22). Here, we analyzed the expression of inflammatory factors in myocardial tissue and serum. ELISA assay showed that Andro treatment at different concentrations decreased the levels of TNF- $\alpha$ (Figure 3B), MCP-1 (Figure 3C), hs-CRP (Figure 3D), and IL-1 $\beta$ (Figure $3 E$ ) in serum. Together, these results indicated that Andro shifted the phenotype macrophage from pro-inflammatory subset to anti-inflammatory subset and ameliorated the expression of inflammatory factors.

\section{Andrographolide inhibited cardiac apoptosis and regulated the protein activities of PPAR $\alpha$ and $N F-\kappa B$}

As shown in Figure $4 A$, the expression of caspase-3 was decreased, compared with control $(\mathrm{P}<0.05)$. However, the levels caspase-3 were reversed after Andro treatment (10 and $50 \mathrm{mg} / \mathrm{kg}$ ). In addition, Figure $4 B$ showed that the 

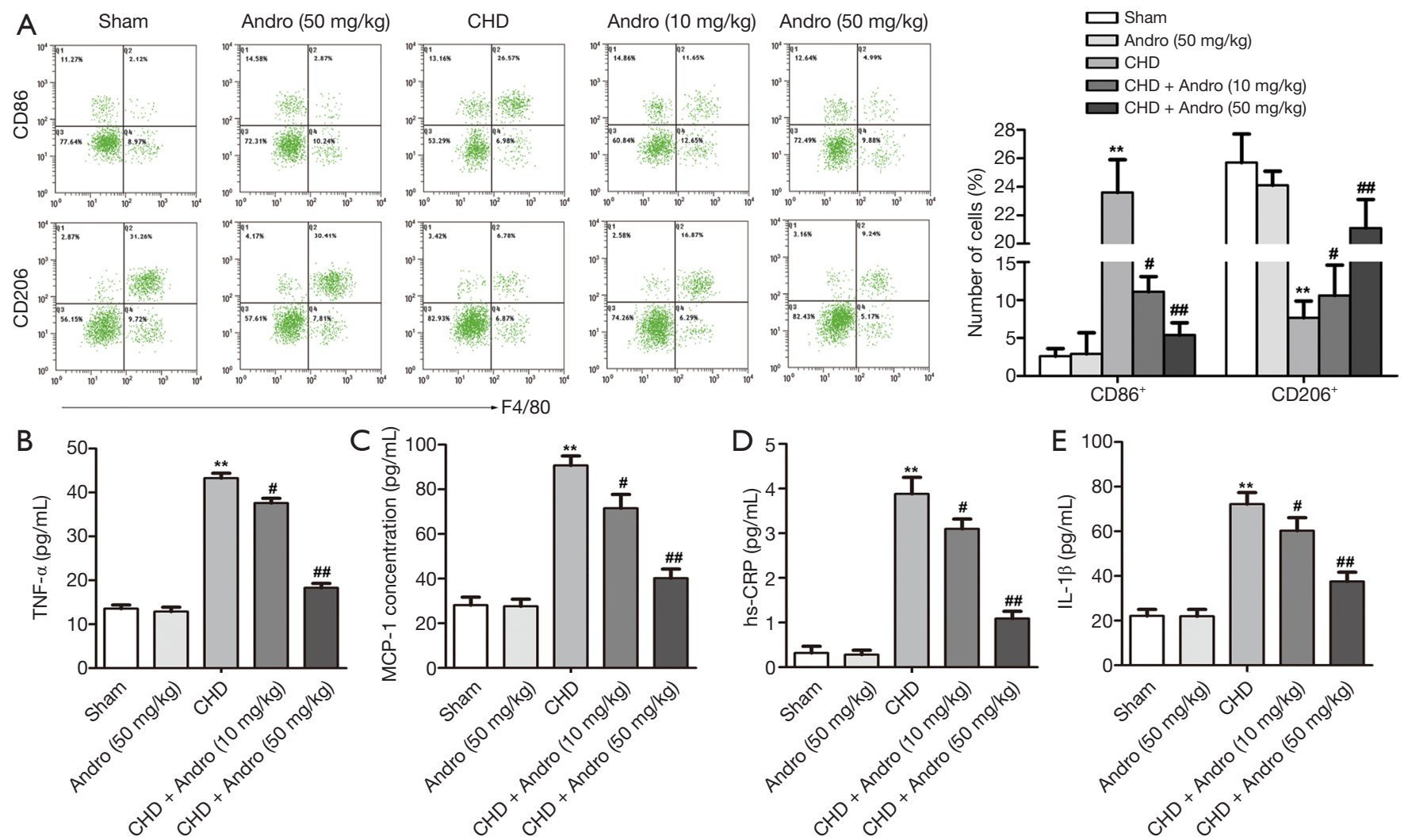

Figure 3 Effect of Andro on myocardial inflammation. (A) The distribution of CD86 and CD206 macrophages was detected by flow cytometry; $(\mathrm{B}, \mathrm{C}, \mathrm{D}, \mathrm{E})$ the inflammatory factor TNF- $\alpha, \mathrm{MCP}-1$, hs-CRP, and IL- $1 \beta$ were as by ELLSA assay. Data values are presented as mean $\pm \mathrm{SD} ; \mathrm{n}=10$. **, $\mathrm{P}<0.01$ vs. sham group; ${ }^{\#}, \mathrm{P}<0.05,{ }^{\# \#}, \mathrm{P}<0.01$ vs. CHD group. Andro, andrographolide; CHD, coronary heart disease.

value of $\mathrm{Bcl}-2 / \mathrm{Bax}$ was increased with Andro treatment. To elucidate the underlying mechanism of Andro, we detected the expression of related proteins by western blot. Our results revealed that PPAR $\alpha$ protein expression was significantly lower while the phosphorylation levels of p65 and I $\kappa \mathrm{B} \alpha$ were remarkably higher in the CHD group compared with the sham group. On the contrary, Andro reversed the change of PPAR $\alpha, \mathrm{p} 65$, and $\mathrm{I} \kappa \mathrm{B} \alpha$ protein (Figure 4C).

\section{Andrographolide attenuated CHD through suppressing PPARa patbway}

To confirm whether PPAR $\alpha$ signaling is involved in Andro's protective effect on CHD, we injected PPAR $\alpha$ antagonist GW6471 (1 nM) into the jugular vein of rats, and rats were randomly separated into five groups: sham group, CHD group, CHD + GW6471 (1 nM) group, and CHD + Andro $(50 \mathrm{mg} / \mathrm{kg})$ group, and GW6471 + Andro group. As shown in Figure $5 A$, the protein level of PPAR $\alpha$ was significantly decreased and the expression of $\mathrm{p}-\mathrm{p} 65$ and $\mathrm{p}-\mathrm{I} \kappa \mathrm{B} \alpha$ were dramatically increased compared with sham, and the addition of GW6471 made this trend more obvious. After GW6471 + Andro treatment, this trend was removed. In addition, we also tested the expression of ET, NO, TXA2, TNF- $\alpha$, MCP- 1 , hs-CRP, and IL- $1 \beta$ by ELISA. The expression of ET, TXA2, TNF- $\alpha$, MCP-1, hs-CRP, and IL-1 $\beta$ were significantly increased, while the expression of NO was dramatically decreased compared with sham group. Andro remarkably decreased the proteins levels of ET, TXA2, TNF- $\alpha$, MCP-1, hs-CRP, and IL- $1 \beta$, while it increased the protein level of NO compared with CHD. GW6471 significantly aggravated the changes in these proteins when compared with CHD. Moreover, GW6471 + Andro treatment weakened the levels of ET, TXA2, TNF- $\alpha$, MCP-1, hs-CRP, and IL- $1 \beta$ and elevated the level of NO compared with the CHD + GW6471 group (Figure $5 B, C, D, E, F, G, H)$. 
A
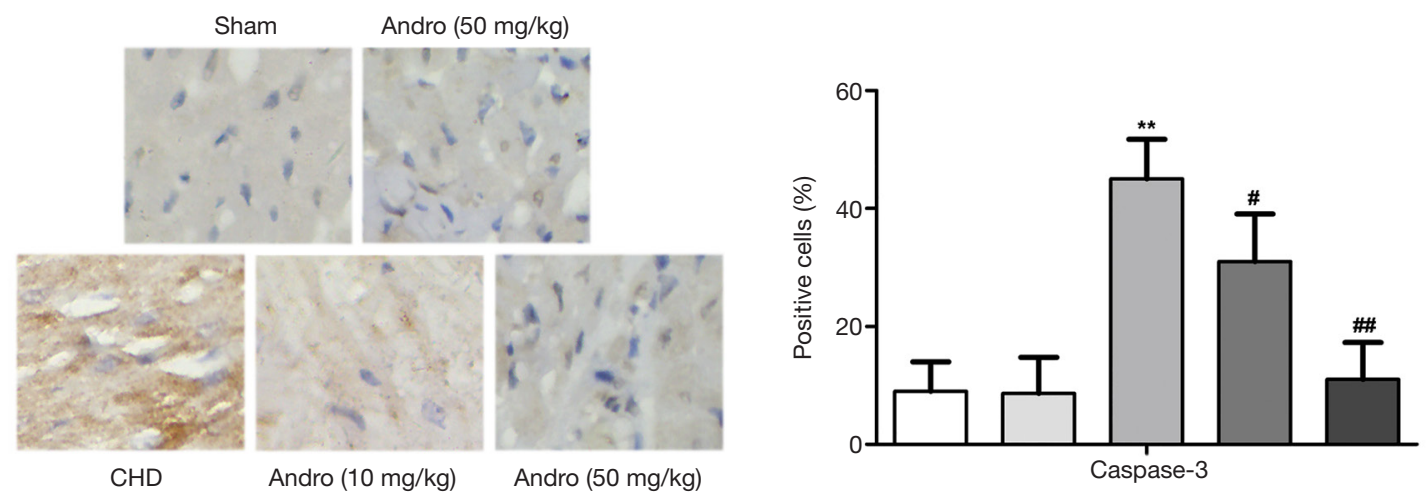

B
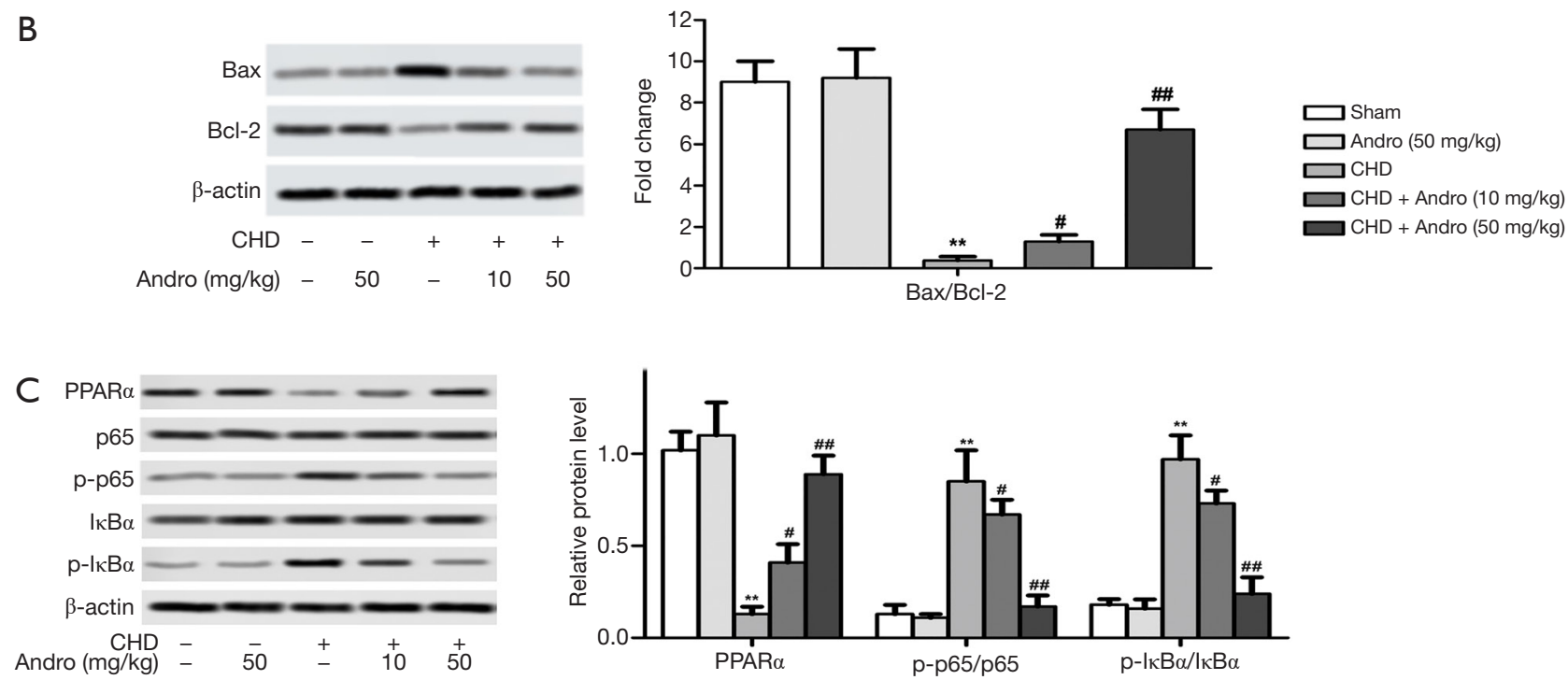

Figure 4 Effect of Andro on cardiac apoptosis and the activation of PPAR $\alpha$ and NF- $\kappa$ B. (A) The expression of caspase-3 was examined by immunohistochemistry, representative graphs were magnified at 200×; (B) the protein levels of Bcl-2 and Bax were examined by WB; (C) the expression of PPAR $\alpha, \mathrm{p} 65, \mathrm{p}-\mathrm{p} 65, \mathrm{I} \kappa \mathrm{B} \alpha$, and $\mathrm{p}-\mathrm{I} \kappa \mathrm{B} \alpha$ as detected by Western. Data values are presented as mean $\pm \mathrm{SD} ; \mathrm{n}=10$. ${ }^{* *}, \mathrm{P}<0.01$ vs. sham group; $, \mathrm{P}<0.05,{ }^{\#}, \mathrm{P}<0.01$ vs. CHD group. Andro, andrographolide; CHD, coronary heart disease.

\section{Discussion}

In recent years, CHD has become a major health problem for the general population. Previous studies have found that an inflammatory response is related to the progression of CHD (23). Consequently, inflammatory factors and endothelial function can be viewed as available indicators for assessing and treating CHD (10). In this study, we explored the therapeutic effect of Andro on CHD mice. The results showed that Andro treatment reduced the serum levels of TNF- $\alpha$, MCP-1, hs-CRP, and L- $1 \beta$, attenuated the endothelial dysfunction, and impeded cardiac apoptosis in CHD rats. Andro therapy improved the CHD process of mouse model by regulating PPAR $\alpha$ and NF- $\mathrm{KB}$ signals.

Endothelial dysfunction is the initial stage of CHD, which is characterized by decreased endothelial-dependent vasodilation, NO synthesis, and vascular motility factor disorders (24). PGI2 and NO have been considered as the two main components of endothelial-derived relaxing factors and play an important role in regulating vascular movement $(21,25)$. In addition, endothelial cells also produce a variety of vasoconstriction factors, such as ET-1 and TXA2, which can stimulate leukocyte adhesion (26) and promote platelet aggregation (27), respectively. Endothelium-derived imbalance can cause endothelial dysfunction. This study showed that Andro significantly promoted the up-regulation of PIG2 and NO and inhibited the expression of ET-1 and TXA2, suggesting that Andro improved microcirculation function by maintaining balance 

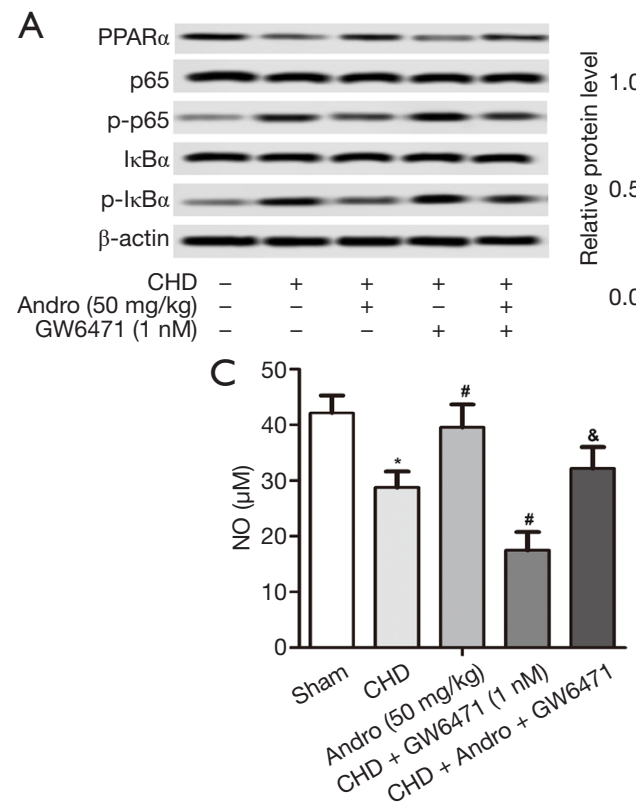

$\mathrm{F}$

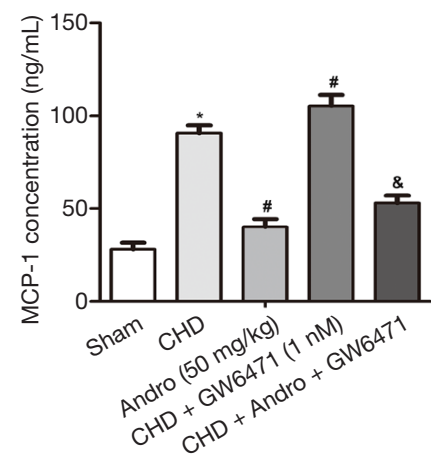

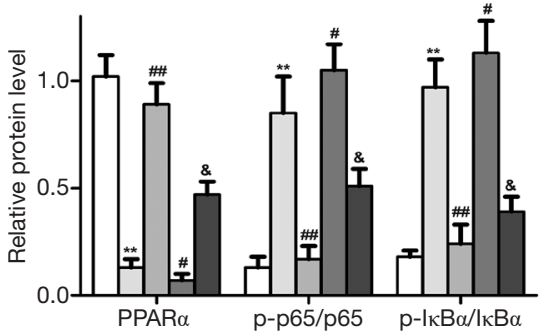
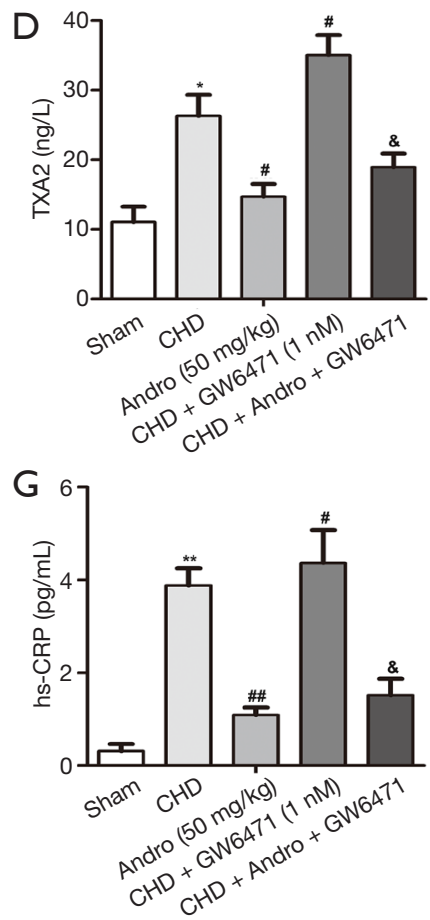
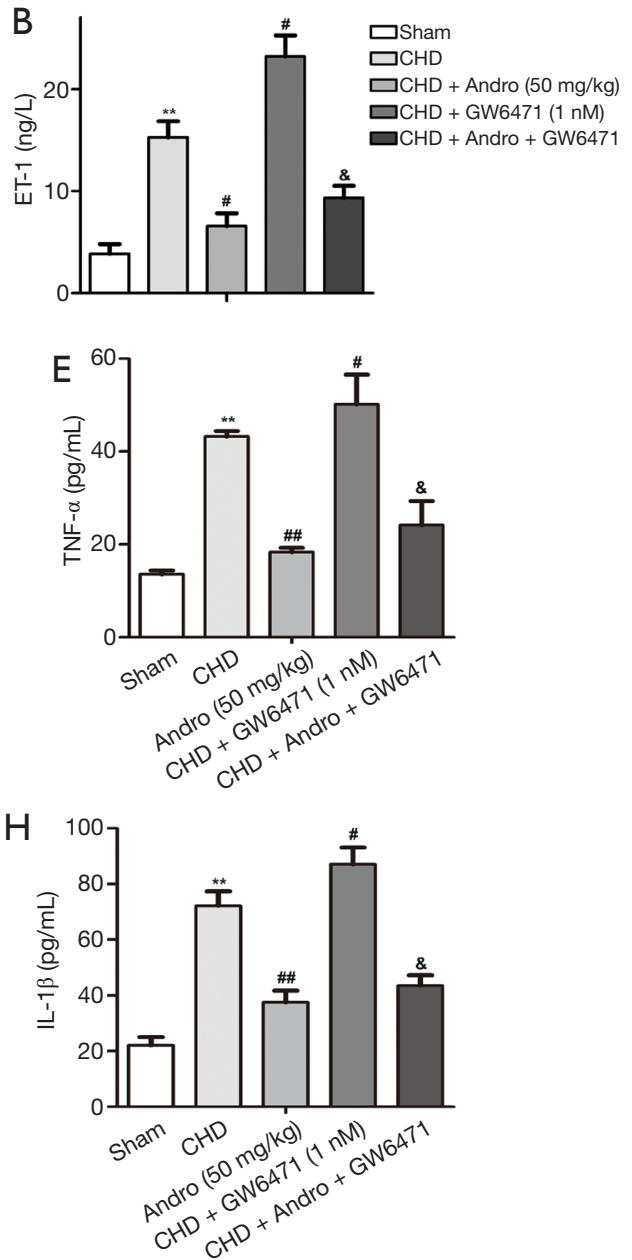

Figure 5 Effect of Andro on CHD in the presence of GW6471. After adding PPAR $\alpha$ antagonist GW6471. (A) The protein levels of PPAR $\alpha$,

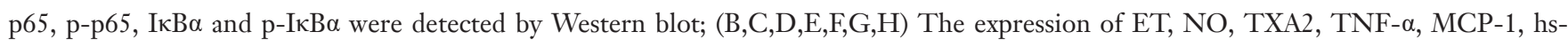
CRP, and IL- $1 \beta$ as detected by ELISA assay. Data values are presented as mean $\pm \mathrm{SD} ; \mathrm{n}=10 .{ }^{*}, \mathrm{P}<0.05$, **, $\mathrm{P}<0.01$ vs. sham group; ${ }^{*}, \mathrm{P}<0.05$, \#, $\mathrm{P}<0.01$ vs. CHD group; ${ }^{*}, \mathrm{P}<0.05$ vs. CHD + GW6471 (1 nM). Andro, andrographolide; NO, nitric oxide; CHD, coronary heart disease.

between endothelial cells.

Inflammation is one of the most common symptoms in patients with acute CHD (28). Li et al. found that transient reduction of TNF- $\alpha$ in children's serum was an important biological indicator for monitoring children with congenital heart disease (29). IL-1 $\beta$ accelerates endothelial permeability and stimulates the release of chemokines, leading to the accumulation of inflammatory cells including neutrophils and macrophages (30). In addition, MCP1 specifically regulates the migration and activation of monocytes and macrophages, affecting the growth and stability of atherosclerotic plaques (31). Particularly, Andro has been shown to have anti-inflammatory effects (15-17).
In line with these studies, we found that Andro switched the polarization of macrophages from pro-inflammatory subset to anti-inflammatory subset, decreased the levels of TNF- $\alpha$, MCP- 1 , and hs-CRP, IL- $1 \beta$, indicating that Andro suppressed myocardial inflammation in rats with CHD.

$\mathrm{NF}-\kappa \mathrm{B}$ is a pleiotropic transcription-inducing factor, and activation of NF- $\mathrm{KB}$ is related to cardiac cell apoptosis and cytokine release (32). I $\kappa$ Bs will rapidly phosphorylate and degrade after activation of NF- $\kappa \mathrm{B}$, leading to the transfer of $\mathrm{p} 50 / \mathrm{p} 65$ heterodimers to the nucleus, stimulating the transcription of multiple target genes, thereby regulating the inflammatory factors VIL-1 (IL-1b, IL-6, and TNF-a) and cytokines/chemokines $(32,33)$. Xia et al. reported 
that Andro prevented allergic lung inflammation in

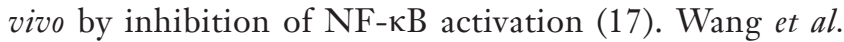
found Andro inhibited NF- $\kappa \mathrm{B}$ activation and attenuated neointimal hyperplasia in arterial restenosis (15).

In this study, our results showed that Andro inhibited the phosphorylation of $\mathrm{p} 65$ and I $\mathrm{KB} \alpha$. PPAR $\alpha$, as a major transcriptional regulator of energy metabolism (34), is expressed in multiple organs such as the liver, kidney, and heart. It is essential for regulating inflammation and angiogenesis. PPAR $\alpha$ levels were reported to be downregulated in animal models or patients with heart failure $(35,36)$. In our rat model, PPAR $\alpha$ levels were significantly downregulated, which is consistent with previous reports. Inhibition or reduction of PPAR $\alpha$ expression may cause a decrease in the ability of cardiomyocytes to oxidize fatty acid substrates, leading to a decrease in ATP during heart failure (37). In addition, in the pig model, PPAR $\alpha$ ligand was found to inhibit the proliferation and migration of endothelial cells and reduce angiogenesis. In addition to generating energy, PPAR $\alpha$ can also regulate apoptosis, which is an important contributing factor to heart failure (38). Due to its cardioprotective effect, PPAR $\alpha$ can be used as a target for the treatment of heart failure. To further explore the regulatory effect of Andro on PPAR $\alpha$, we added the PPAR $\alpha$ antagonist GW6471. Compared with the CHD group, GW6471 significantly inhibited the expression of PPAR $\alpha$, while Andro and GW6471 co-treatment increased the protein level of PPAR $\alpha$. In parallel fashion, we also found that GW6471 exacerbated the inflammatory response in a rat model, while Andro eliminated the GW6471 stimulation of inflammation.

In conclusion, our study demonstrated the protective effect of Andro on CHD, which was mediated via PPAR and NF- $\mathrm{KB}$ signaling pathways. Furthermore, as Andro alleviated myocardial injury, inhibited cardiac apoptosis, mitigated endothelial dysfunction and fibrinolytic activity, and ameliorated myocardial inflammation, it may thus be a candidate for the treatment of CHD.

\section{Acknowledgments}

Funding: None.

\section{Footnote}

Reporting Checklist: The authors have completed the ARRIVE reporting checklist. Available at http://dx.doi. org/10.21037/apm-20-960

Data Sharing Statement: Available at http://dx.doi. org/10.21037/apm-20-960

Conflicts of Interest: All authors have completed the ICMJE uniform disclosure form (available at http://dx.doi. org/10.21037/apm-20-960). The authors have no conflicts of interest to declare.

Ethical Statement: The authors are accountable for all aspects of the work in ensuring that questions related to the accuracy or integrity of any part of the work are appropriately investigated and resolved. All animal protocols were conducted in accordance with the institute guidelines for the care and use of animal, and approved by the ethics committees of Chengdu University of Traditional Chinese Medicine (No. SYXK-20200046).

Open Access Statement: This is an Open Access article distributed in accordance with the Creative Commons Attribution-NonCommercial-NoDerivs 4.0 International License (CC BY-NC-ND 4.0), which permits the noncommercial replication and distribution of the article with the strict proviso that no changes or edits are made and the original work is properly cited (including links to both the formal publication through the relevant DOI and the license). See: https://creativecommons.org/licenses/by-nc-nd/4.0/.

\section{References}

1. Agarwal MA, Garg L, Lavie CJ, Reed GL, Khouzam RN. Impact of family history of coronary artery disease on in-hospital clinical outcomes in ST-segment myocardial infarction. Ann Transl Med 2018;6:3.

2. Gao R, Yang $Y$, Han $Y$, et al. Bioresorbable vascular scaffolds versus metallic stents in patients with coronary artery disease: ABSORB China trial. J Am Coll Cardiol 2015;66:2298-309.

3. Fan FF, Xu Q, Sun Q, et al. Assessment of the reporting quality of randomized controlled trials on treatment of coronary heart disease with traditional chinese medicine from the Chinese journal of integrated traditional and Western medicine: a systematic review. PLoS One 2014;9:e86360.

4. Tully PJ, Baumeister H. Collaborative care for comorbid depression and coronary heart disease: A systematic review and meta analysis of randomised controlled trials. BMJ 
Open 2015;5:e009128.

5. Berndt N, Bolman C, Froelicher ES, et al. Effectiveness of a telephone delivered and a face to face delivered counseling intervention for smoking cessa- tion in patients with coronary heart disease: A 6 month follow up. J Behav Med 2014;37:709-24.

6. Zhu L, Chen T, Ye W, et al. Circulating miR-182-5p and miR-5187-5p as biomarkers for the diagnosis of unprotected left main coronary artery disease. J Thorac Dis 2019;11:1799-808.

7. Bollati V, Angelici L, Rizzo G, et al. Microvesicle associated microRNA expression is altered upon particulate matter exposure in healthy workers and in A549 cells. J Appl Toxicol 2015;35:59-67.

8. Azambuja MI. Inflammation as the cause of coronary heart disease. Lancet Infect Dis 2010;10:142-3.

9. Sukumaran V, Tsuchimochi H, Sonobe T, et al. Liraglutide treatment improves the coronary microcirculation in insulin resistant Zucker obese rats on a high salt diet. Cardiovasc Diabetol 2020;19:24.

10. Derosa G, Bonaventura A, Bianchi L, et al. A randomized, placebo-controlled study on the effects of a nutraceutical combination of red yeast rice, silybum marianum and octasonol on lipid profile, endothelial and inflammatory parameters. J Biol Regul Homeost Agents 2014;28:317-24.

11. Kuravi SJ, McGettrick HM, Satchell SC, et al. Podocytes regulate neutrophil recruitment by glomerular endothelial cells via IL 6 mediated crosstalk. J Immunol 2014;193:234-43.

12. Roxas M, Jurenka J. Colds and influenza: a review of diagnosis and conventional, botanical, and nutritional considerations. Altern Med Rev 2007;12:25-48.

13. Zhou J, Lu GD, Ong CS, et al. Andrographolide sensitizes cancer cells to TRAIL-induced apoptosis via p53-mediated death receptor 4 up-regulation. Mol Cancer Ther 2008;7:2170-80.

14. Negi AS, Kumar JK, Luqman S, et al. Recent advances in plant hepatoprotectives: A chemical and biological profile of some important leads. Med Res Rev 2008;28:746-72.

15. Wang YJ, Wang JT, Fan QX, et al. Andrographolide inhibits NF-kB activation and attenuates neointimal hyperplasia in arterial restenosis. Cell Res 2007;17:933-41.

16. Chiou WF, Chen CF, Lin JJ. Mechanisms of suppression of inducible nitric oxide synthase (iNOS) expression in RAW 264.7 cells by andrographolide. Br J Pharmacol 2000;129:1553-60.
17. Xia YF, Ye BQ, Li YD, et al. Andrographolide attenuates inflammation by inhibition of NF-kappa B activation through covalent modification of reduced cysteine 62 of p50. J Immunol 2004;173:4207-17.

18. Gonzalez L, Yu P, Trigatti B. Mouse models of coronary artery atherosclerosis. J Cardiovasc Disord 2016;3:1021.

19. Liang Ershun, Liu X, Du Z, et al. Andrographolide ameliorates diabetic cardiomyopathy in mice by blockage of oxidative damage and NF- $\mathrm{Nm}$-mediated inflammation. Oxid Med Cell Longev ongevell Long

20. Lazzè MC, Pizzala R, Perucca P, et al. Anthocyanidins decrease endothelin-1 production and increase endothelial nitric oxide synthase in human endothelial cells. Mol Nutr Food Res 2006;50:44-51.

21. Smith JB, Araki H, Lefer AM. Thromboxane A2, prostacyclin and aspirin: effects on vascular tone and platelet aggregation, Circulation 1980;62:V19-V25.

22. Werba JP, Veglia F, Amato M, et al. Patients with a history of stable or unstable coronary heart disease have different acute phase responses to an inflammatory stimulus. Atherosclerosis 2008;196:835-40.

23. Li D, Wei W, Ran X, et al. Lipoprotein- associated phospholipase A2 and risks of coronary heart disease and ischemic stroke in the general population: A systematic review and meta-analysis. Clin Chim Acta 2017;471:38-45.

24. Dusting GJ. Nitric oxide in coronary artery disease: Roles in atherosclerosis, myocardial reperfusion and heart failure. EXS 1996;76:33-55.

25. Baptista J, Teles RC, da Silva PC, et al. Five year clinical results of coronary angioplasty with drug eluting stents. National initiative in strategic innovation, iNOS. Rev Port Cardiol 2010;29:243-51.

26. Thengchaisri N, Hein TW, Ren Y, et al. Endothelin-1 impairs coronary arteriolar dilation: Role of p38 kinasemediated superoxide production from NADPH oxidase. J Mol Cell Cardiol 2015;86:75-84.

27. DeFilippis AP, Oloyede OS, Andrikopoulou E, et al. Thromboxane $\mathrm{A}(2)$ generation, in the absence of platelet COX-1 activity, in patients with and without atherothrombotic myocardial infarction. Circ J 2013;77:2786-92.

28. Steptoe A, Wikman A, Molloy GJ, et al. Inflammation and symptoms of depression and anxiety in patients with acute coronary heart disease. Brain Behav Immun 2013;31:183-8.

29. Li Z, Wu JC, Sheikh AY, et al. Differentiation, survival, and function of embryonic stem cell derived endothelial cells for ischemic heart disease. Circulation 2007;116:I46-54. 
30. Swirski FK, Nahrendorf M. Leukocyte behavior in atherosclerosis, myocardial infarction, and heart failure. Science 2013;339:161-6.

31. Inoshima I, Kuwano K, Hamada N, et al. Anti-monocyte chemoattractant protein-1 gene therapy attenuates pulmonary fibrosis in mice. Am J Physiol Lung Cell Mol Physiol 2004;286:L1038-44.

32. Yang L, Guo H, Li Y, et al. Oleoylethanolamide exerts anti-inflammatory effects on LPS-induced THP-1 cells by enhancing PPARa signaling and inhibiting the NF-kB and ERK1/2/AP-1/STAT3 pathways. Sci Rep 2016;6:34611

33. Lawrence T, Gilroy DW, Colville-Nash PR, et al. Possible new role for NF-kappaB in the resolution of inflammation. Nat Med 2001;7:1291-7.

34. Khuchua Z, Glukhov AI, Strauss AW, et al. Elucidating the Beneficial Role of PPAR Agonists in Cardiac Diseases. Int J Mol Sci 2018. doi: 10.3390/ijms19113464.

Cite this article as: Shu J, Huang R, Tian Y, Liu Y, Zhu R, Shi G. Andrographolide protects against endothelial dysfunction and inflammatory response in rats with coronary heart disease by regulating PPAR and NF- $\mathrm{KB}$ signaling pathways. Ann Palliat Med 2020;9(4):1965-1975. doi: 10.21037/apm-20-960
35. Kasai T, Miyauchi K, Yokoyama T, et al. Efficacy of peroxisome proliferative activated receptor (PPAR)-alpha ligands, fenofibrate, on intimal hyperplasia and constrictive remodeling after coronary angioplasty in porcine models. Atherosclerosis 2006;188:274-80.

36. Cuzzocrea S, Mazzon E, Di Paola R, et al. The role of the peroxisome proliferator-activated receptoralpha (PPARalpha) in the regulation of acute inflammation. J Leukoc Biol 2006;79:999-1010.

37. Lai L, Leone TC, Keller MP, et al. Energy metabolic reprogramming in the hypertrophied and early stage failing heart: a multisystems approach. Circ Heart Fail 2014;7:1022-31.

38. Kuo CY, Chiu YC, Lee AY, et al. Mitochondrial Lon protease controls ROS-dependent apoptosis in cardiomyocyte under hypoxia. 